\title{
High Dynamics of Vapor Adsorption in Ordered Mesoporous Carbon CMK-5: A SAXS Study
}

\author{
W. Schmidt, ${ }^{1, *}$ H. Amenitsch ${ }^{2}$ \\ ${ }^{1}$ Max-Planck-Institut für Kohlenforschung, Kaiser-Wilhelm-Platz 1, 45470 Mülheim an der \\ Ruhr, Germany \\ ${ }^{2}$ Institut für Anorganische Chemie, TU Graz, Stremayrgasse 9/IV, 8010 Graz, Austria
}

Supplementary Information 


\section{CALCULATIONS}

\section{Determination of Invariant $Q$}

For calculating the invariant,

$$
Q=\frac{1}{2 \pi^{2}} \int_{0}^{\infty} I(q) q^{2} d q
$$

the intensities multiplied with $q^{2}$ must be integrated over the complete $q$ range from $q=0$ to infinite q. In the experiment, for practical reasons, data are measured only in a limited q range with a lower $q$ value, $q_{\min }$, to an upper limit,$q_{\max }$. In order to assess $Q$ over the entire $q$ range, intensities at $q<q_{\min }$ and $q>q_{\max }$ must be approximated. We have used a power law, assuming that for large $q$ values the intensity changes with $q^{-4}$ (Porod). At sufficiently high $q$, a plot of the measured intensities versus $q^{-4}$ yields a linear plot that fulfills the equation:

$$
I=\frac{A}{q^{4}}+B
$$

where $A$ is a constant related to the surface area of the material and $B$ the experimental background. Figure C1 shows this exemplarily for one data set.

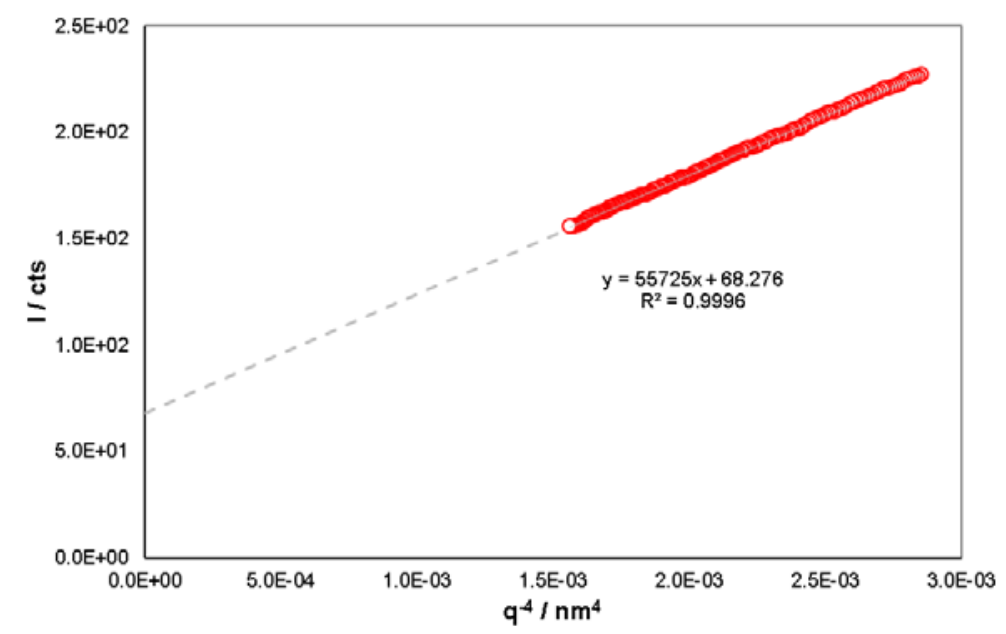

Figure C1. Intensities of the upper 163 data points plotted versus $\mathrm{q}^{-4}$. 
The intensities in the range $q>q_{\max }$ are then extrapolated with eq. $C 1$ using the determined values for $A$ and $B$. Since data cannot be calculated up to infinity, the extrapolation is stopped when intensities get sufficiently small (here $\left.I<\left(/\left(q_{\mathrm{min}}\right) / 100\right)\right)$.

The Guinier formula

$$
I=I(0) \exp \left(-\frac{q^{2} R_{g}^{2}}{3}\right) \text { or } \ln (I)=\ln (I(0))-\left(\frac{q^{2} R_{g}^{2}}{3}\right) \quad \text { (eq. C2) }
$$

was used for calculating the missing intensities below $q_{\min }$. In this formula $I(0)$ denotes the intensity at $q=0$, and $R_{g}$ is a radius of gyration. First, the data (background-corrected) very next to $q_{\min }$ were plotted as $\ln (I)$ versus $q^{2}$ resulting in a linear plot with slope $-R_{g} / 3$ and axis intercept $I(0)$ as shown in Figure C2.

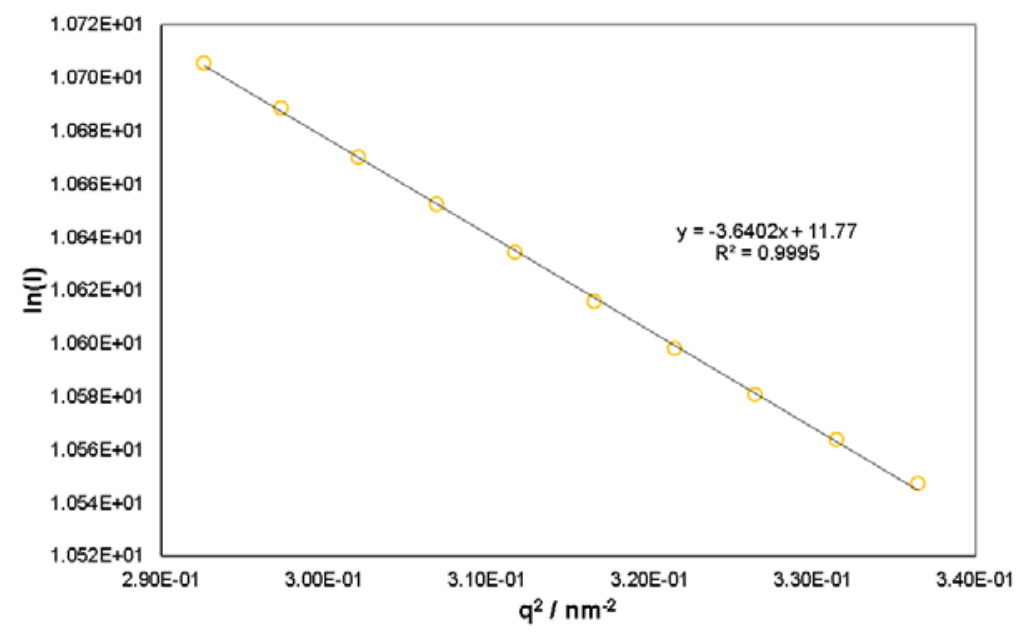

Figure C2. Intensities of 10 data points next to $q_{\min }$ plotted as $\ln (I)$ versus $q^{2}$.

The linear fit yields $R_{g}$ and $I(0)$ and the intensities below $q_{\min }$ then were extrapolated with eq. $C 2$ down to $q=0$ using these values.

After subtracting the background $B$ also from the measured intensities and from the extrapolated intensities at high $q$, all three ranges can be combined as shown in Figure C3. The combined background-corrected curve is then used for calculating the invariant $Q$ according to eq. 1. 


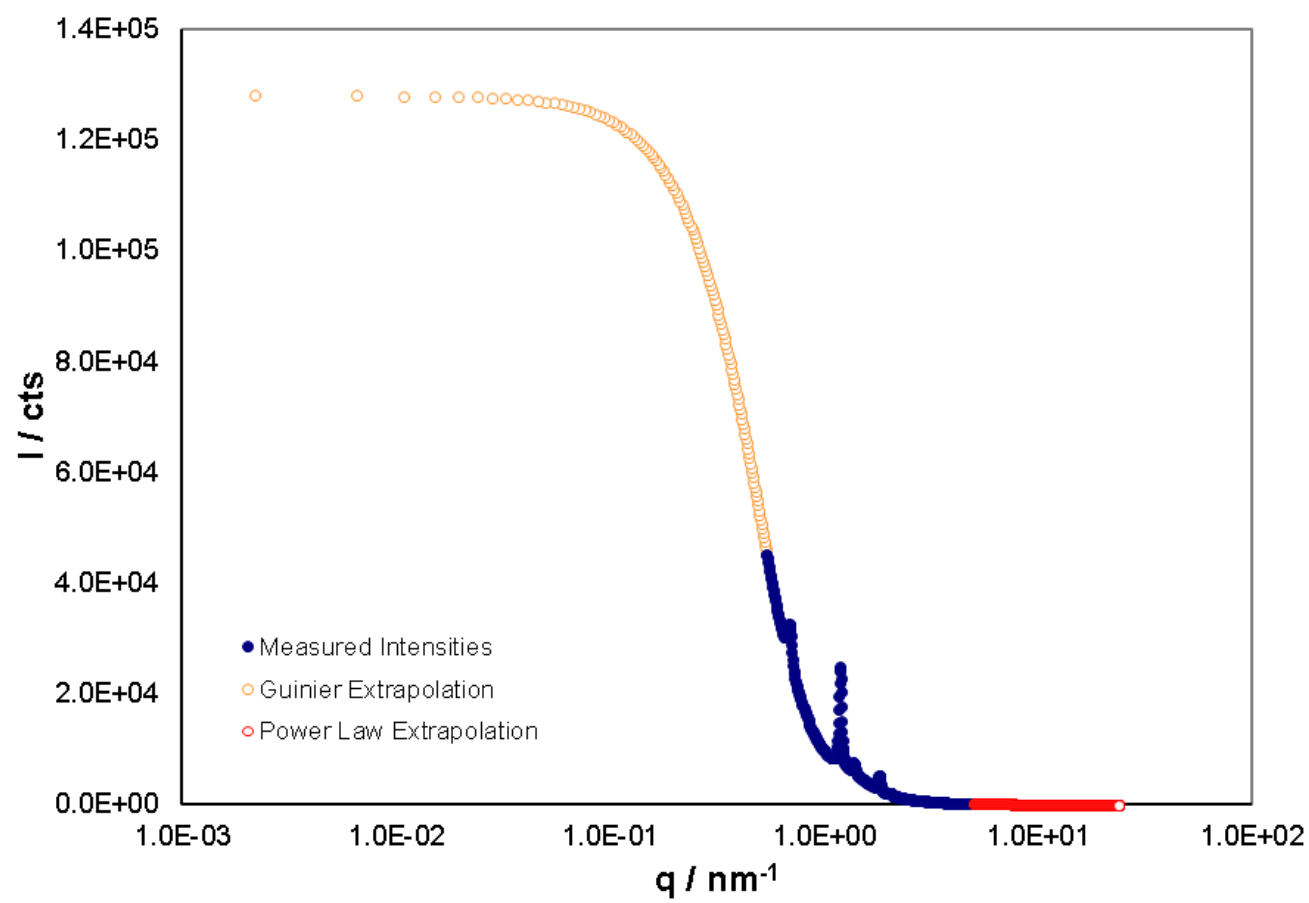

Figure C3. Measured and approximated intensities combined in one plot.

Finally, the background $B$ is also subtracted from the measured intensities, and all three ranges can be combined as shown in Figure $\mathrm{C} 3$. The combined background-corrected curve is then used for calculating the invariant $Q$ according to eq. 1 .

\section{Determination of Adsorption Isotherm from Invariants}

In the following the abbreviations below will be used:

$Q=$ invariant of the scattering intensity

$\Delta \rho_{e}=$ electron density difference between empty pores and pore walls $\Delta \rho_{f}=e$ electron density difference between filled pores and pore walls

$V_{\text {tot }}=$ total pore volume

$V_{e}=$ volume of empty pores

$V_{f}=$ volume of filled pores 
$V_{\text {ads }}(r e l)=$ relative volume adsorbed

In a first approximation, the invariant $Q$ (see eq. 1) is proportional to the following sum

$$
Q=2 \pi^{2}\left(\left(\Delta \rho_{e}\right)^{2} V_{e}+\left(\Delta \rho_{f}\right)^{2} V_{f}\right) \quad \text { (eq.C3) }
$$

The total pore volume $V_{\text {tot }}$ is the sum of $V_{e}$ and $V_{f}$

$$
V_{\text {tot }}=V_{e}+V_{f}
$$

If all pores are empty $V_{f}=0$ and $V_{e}=V_{\text {tot }}$ and the respective invariant $Q_{e}$ calculates

$$
Q_{e}=2 \pi^{2}\left(\Delta \rho_{e}\right)^{2} V_{t o t}
$$

and consequently

$$
\left(\Delta \rho_{e}\right)^{2}=\frac{Q_{e}}{2 \pi^{2} V_{t o t}}
$$

If all pores are filled $V_{e}=0$ and $V_{f}=V_{\text {tot }}$ and the respective invariant $Q_{f}$ calculates

$$
Q_{f}=2 \pi^{2}\left(\Delta \rho_{f}\right)^{2} V_{t o t}
$$

and consequently

$$
\left(\Delta \rho_{f}\right)^{2}=\frac{Q_{f}}{2 \pi^{2} V_{t o t}} \quad \text { (eq.C5) }
$$

We thus can calculate exact values for $\left(\Delta \rho_{e}\right)^{2}$ and $\left(\Delta \rho_{f}\right)^{2}$ and substitution of (eq. C4)and (eq. C5) in (eq. C3) yields

$$
Q=2 \pi^{2}\left(\frac{Q_{e}}{2 \pi^{2} V_{t o t}} V_{e}+\frac{Q_{f}}{2 \pi^{2} V_{t o t}} V_{f}\right)
$$

or

$$
Q=\frac{Q_{e}}{V_{t o t}} V_{e}+\frac{Q_{f}}{V_{t o t}} V_{f}
$$

With $V_{e}=V_{\text {tot }}-V_{f}$ we get

$$
Q=\frac{Q_{e}}{V_{t o t}}\left(V_{t o t}-V_{f}\right)+\frac{Q_{f}}{V_{t o t}} V_{f}
$$

or

$$
Q=Q_{e}-\frac{Q_{e}}{V_{t o t}} V_{f}+\frac{Q_{f}}{V_{t o t}} V_{f}
$$

and

$$
Q=Q_{e}+\frac{V_{f}}{V_{t o t}}\left(Q_{f}-Q_{e}\right)
$$


Reorganization of the formula to $V_{f}$ yields

$$
V_{f}=V_{t o t} \frac{Q-Q_{e}}{Q_{f}-Q_{e}}
$$

and the relative volume of adsorbed vapor $V_{\text {ads }}(r e l)=\left(V_{f} / V_{\text {tot }}\right)$ calculates as

$$
V_{a d s}(r e l)=\frac{V_{f}}{V_{t o t}}=\frac{Q-Q_{e}}{Q_{f}-Q_{e}} \quad(\text { eq. } 2)
$$

\section{TABLES}

\begin{tabular}{|c|c|c|}
\hline & & $\alpha_{h k}$ \\
\hline-1 & 0 & \\
\hline-1 & 1 & \\
\hline 0 & -1 & \\
\hline 0 & 1 & \\
\hline 1 & -1 & \\
\hline 1 & 0 & \\
\hline-2 & 1 & \\
\hline-1 & -1 & \\
\hline-1 & 2 & \\
\hline 1 & -2 & \\
\hline 1 & 1 & \\
\hline 2 & -1 & \\
\hline-2 & 0 & \\
\hline-2 & 2 & \\
\hline 0 & -2 & \\
\hline 0 & 2 & \\
\hline 2 & -2 & \\
\hline 2 & 0 & \\
\hline-3 & 1 & \\
\hline-3 & 2 & \\
\hline-2 & -1 & \\
\hline-2 & 3 & \\
\hline-1 & -2 & \\
\hline-1 & 3 & \\
\hline 1 & -3 & \\
\hline 1 & 2 & \\
\hline
\end{tabular}

Table S1. Phase factors $\alpha_{h k}$ for $F_{h k}$ used in this work: 


$\begin{array}{rrr}2 & -3 & 1 \\ 2 & 1 & 1 \\ 3 & -2 & 1 \\ 3 & -1 & 1 \\ -3 & 0 & 1 \\ -3 & 3 & 1 \\ 0 & -3 & 1 \\ 0 & 3 & 1 \\ 3 & -3 & 1 \\ 3 & 0 & 1 \\ -4 & 2 & 1 \\ -2 & -2 & 1 \\ -2 & 4 & 1 \\ 2 & -4 & 1 \\ 2 & 2 & 1 \\ 4 & -2 & 1 \\ -4 & 1 & -1 \\ -4 & 3 & -1 \\ -3 & -1 & -1 \\ -3 & 4 & -1 \\ -1 & -3 & -1 \\ -1 & 4 & -1 \\ 1 & -4 & -1 \\ 1 & 3 & -1 \\ 3 & -4 & -1 \\ 3 & 1 & -1 \\ 4 & -3 & -1 \\ 4 & -1 & -1 \\ -4 & 0 & -1 \\ -4 & 4 & -1 \\ 0 & -4 & -1 \\ 0 & 4 & -1 \\ 4 & -4 & -1 \\ 4 & 0 & -1\end{array}$




\section{FIGURES}

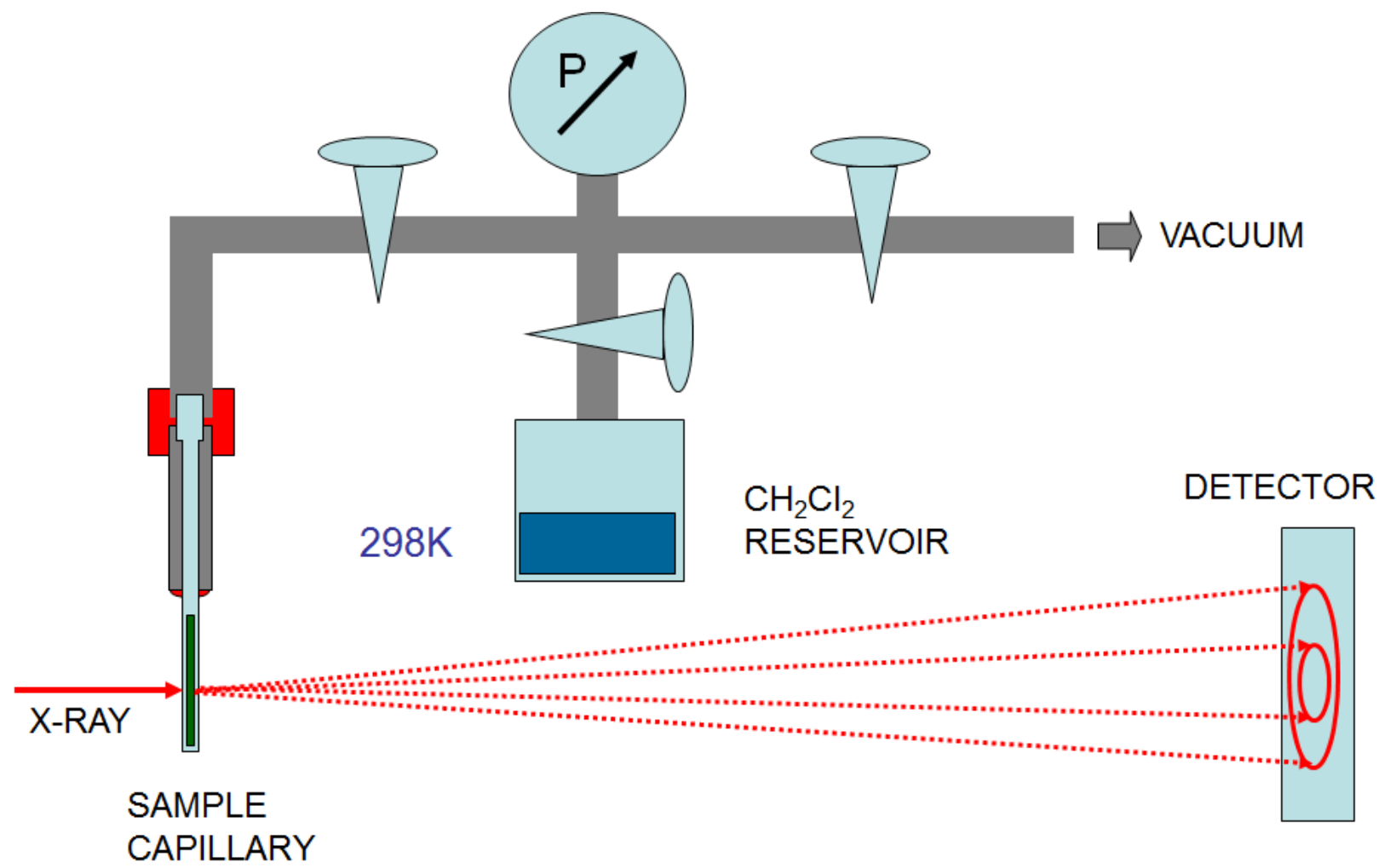

Figure S1. Experimental setup for in-situ SAXS measurements during vapor adsorption at room temperature. 

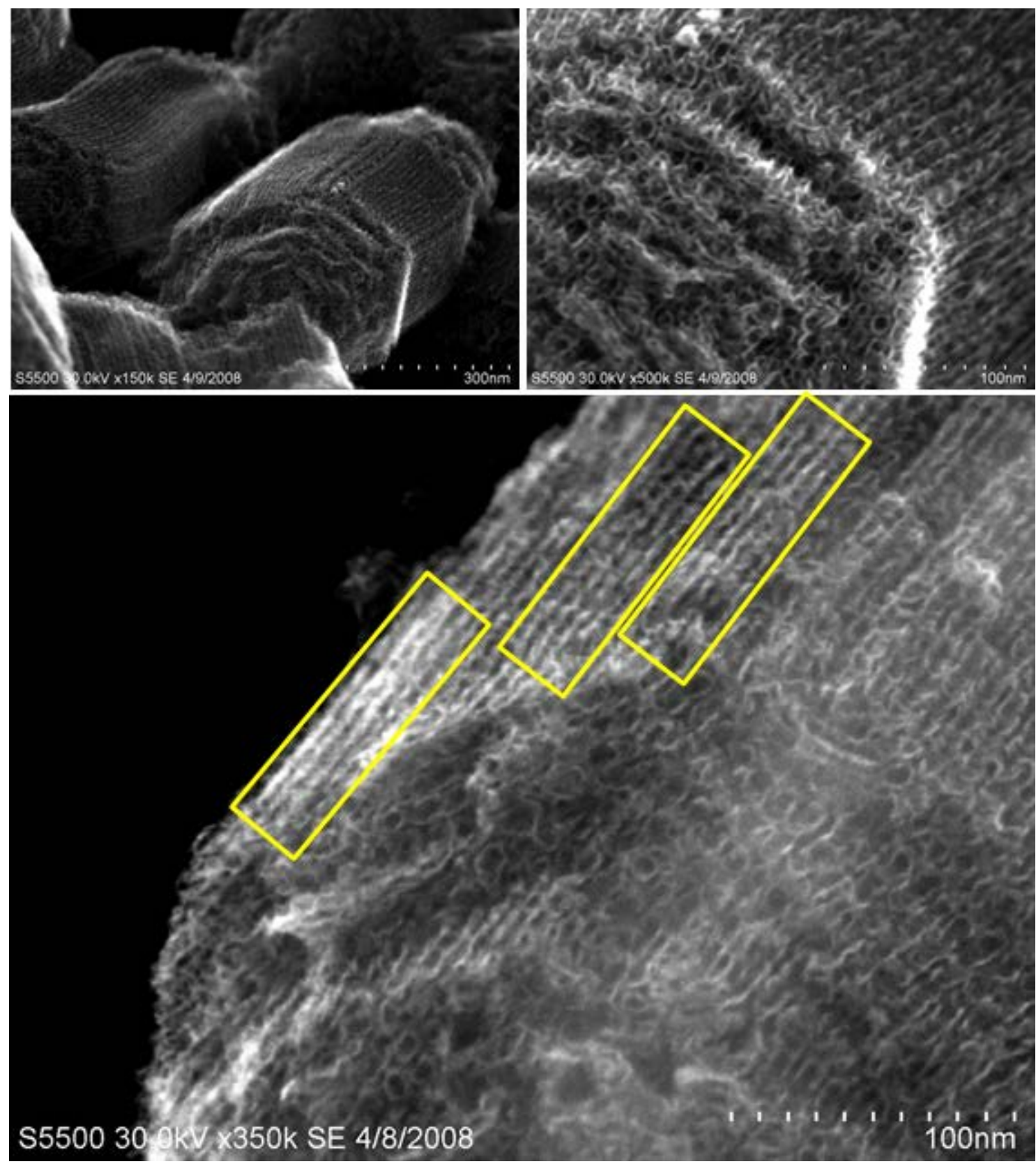

Figure S2. STEM images of the CMK-5 material. The marked areas show open tubes in which certain undulation of the pore diameters is visible. 

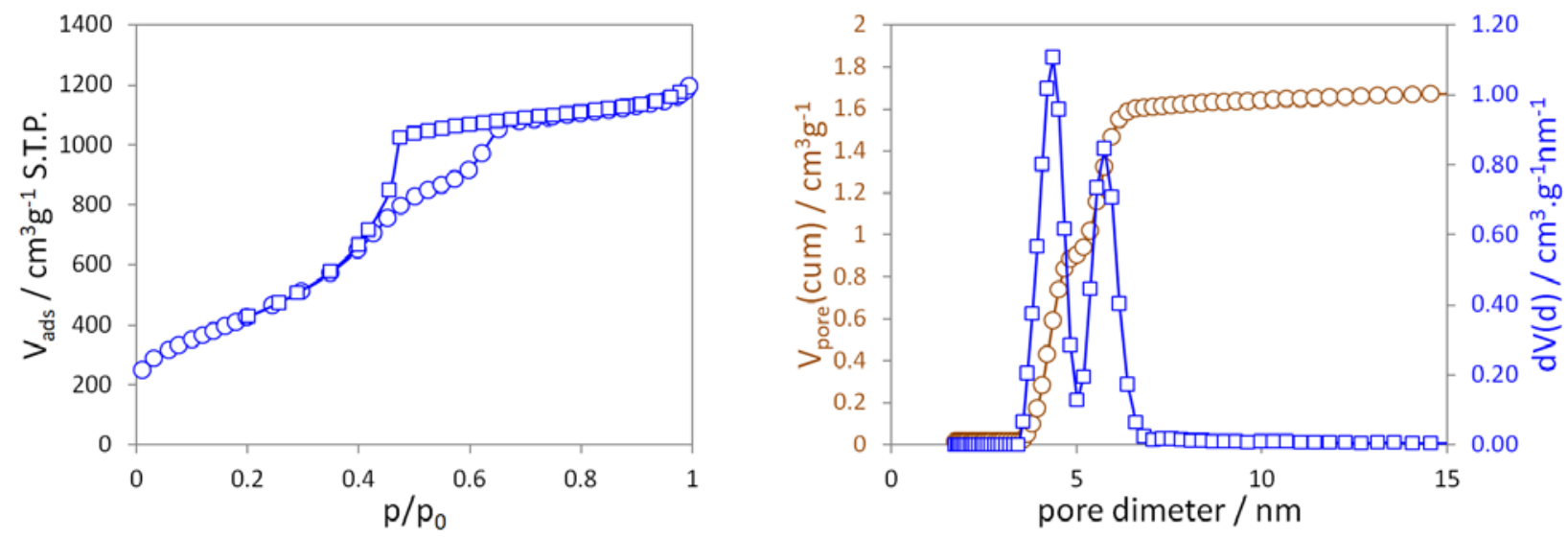

Figure S3. $\mathrm{N}_{2}$ sorption isotherm (left) of the CMK-5 with pore size distribution (right) as calculated with the QSDFT kernel for $\mathrm{N}_{2}$ adsorption on carbon with cylinder pores at 77K of the Quantachrome ASiQWin 4.0 software package.

The adsorption isotherm shows two defined steps indicating two types of mesopores with different pore sizes as reflected in the pore sizes distribution. The desorption branch of the isotherm indicates bore blocking, causing a delayed desorption. Reason for the delayed desorption is most likely undulation of pore diameters within the carbon tubes. This can be seen from HRSEM (STEM) images as shown in Figure S2. The carbon tubes on the surface of the particles are half open in the marked areas (yellow frames). They show no uniform diameters but contain also narrow passages, resulting in a certain variation of pore diameters (undulated pores). Such narrower pore diameters in parts of the tubes are most likely present throughout the complete carbon material. They cause pore restrictions within the larger pores which then result in delayed desorption (ink-bottle pores). SAXS 'sees' that variation of pore diameters as a broadening of averaged electron density distribution, i.e., broader distributions of pore diameters and pore wall thicknesses. 


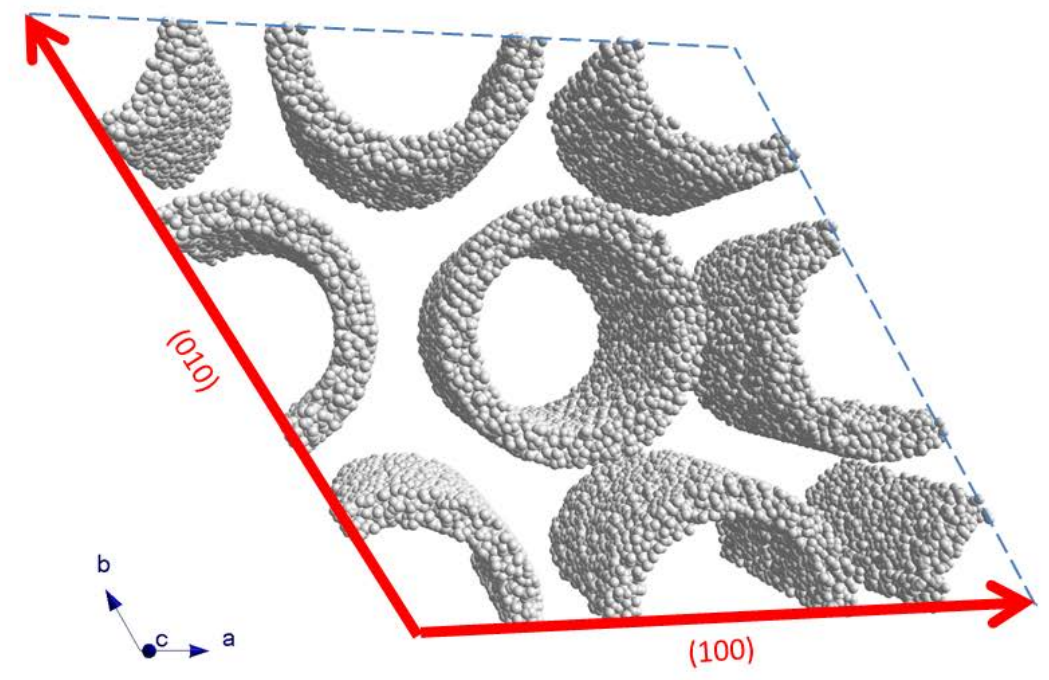

Figure S4. Model of periodic hexagonal arrangement of carbon nanotubes in CMK-5.
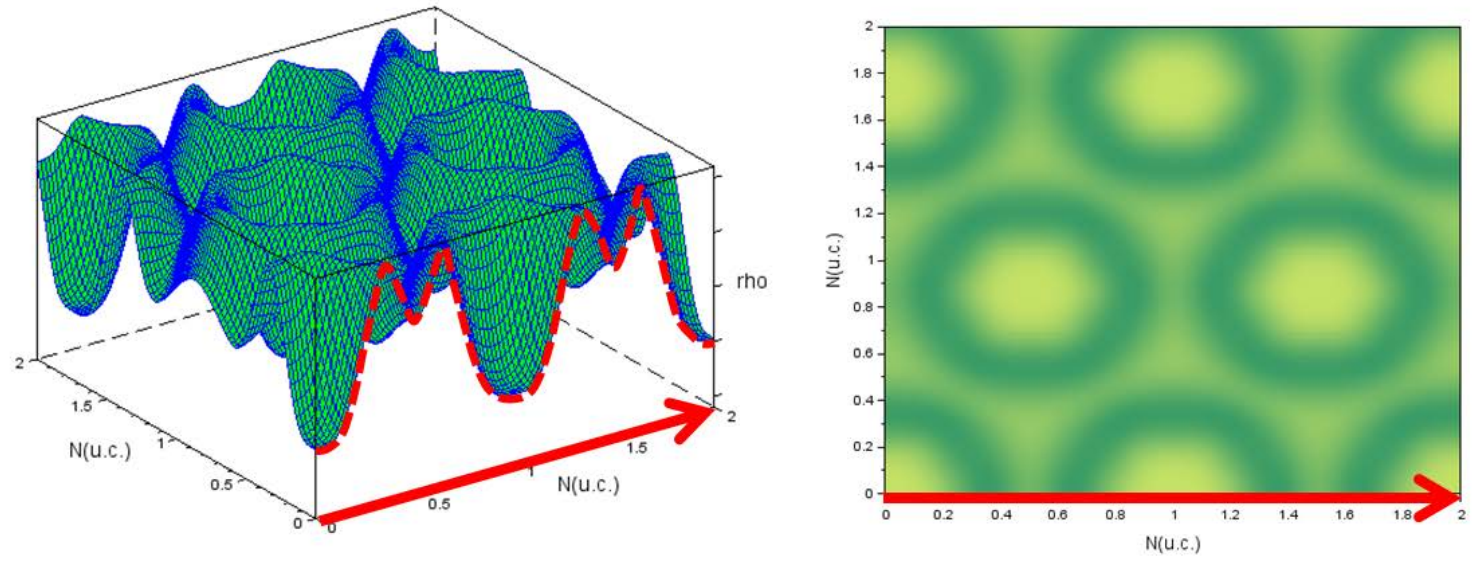

Figure S5. Electron density maps in 3D (left) and 2D (right) representation. The red arrow shows the (100) direction of the electron density line scans. The dotted red line (left) indicates the line scans along (100) over the lengths of two unit cells. 


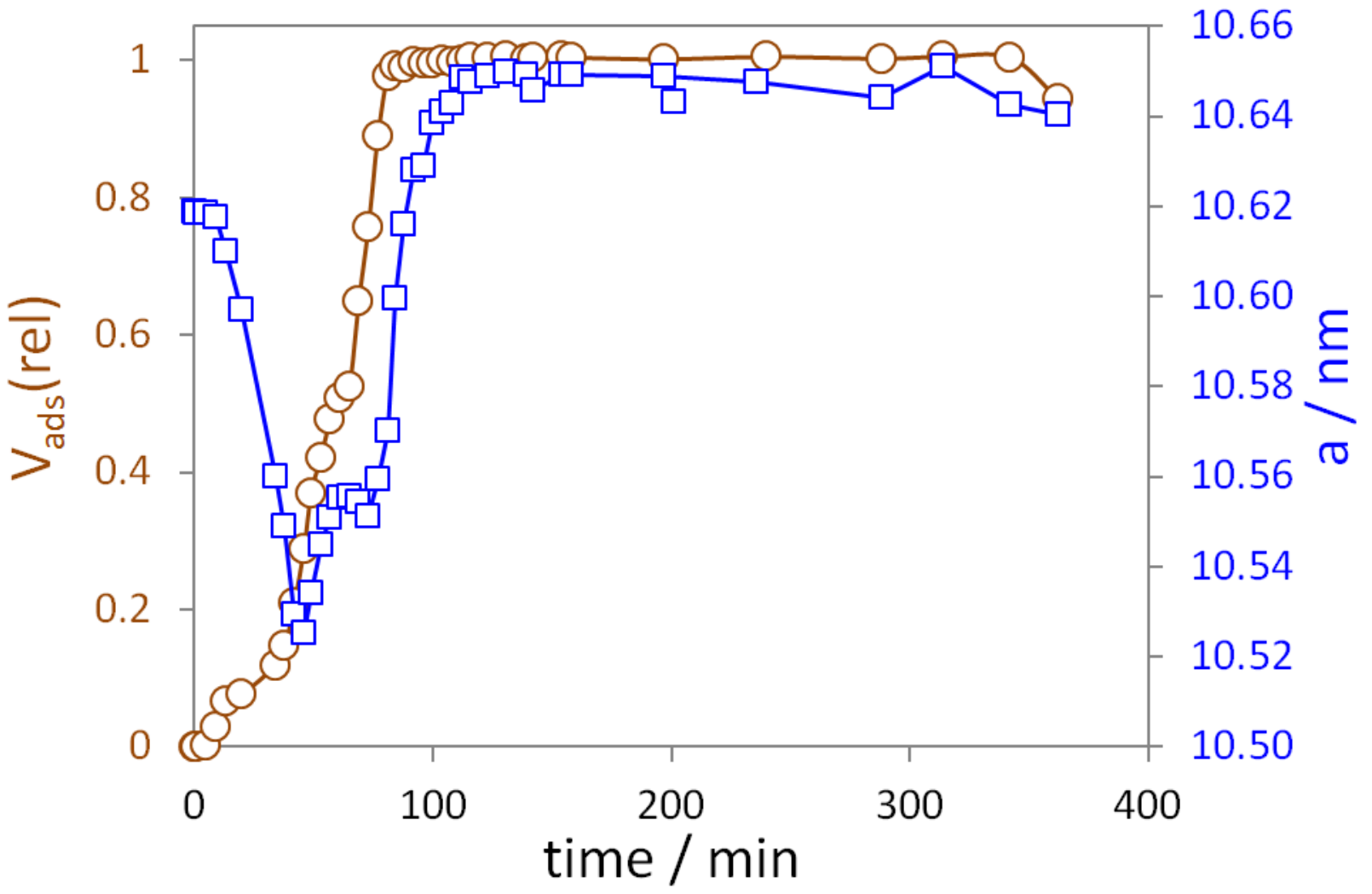

Figure S6. Changes of lattice parameter $a$ and relative volume adsorbed (calculated from invariants $Q$ ) over the whole period of the experiment. For easier visibility, only data from the first 150 min are shown in the main text. As can be seen from the data, adsorption is completed after about $120 \mathrm{~min}$. No significant changes of volume adsorbed and lattice parameter are observed after 120 min.

Figure S7. The figures on the next two pages illustrate the results from the analyses of the SAXS data. The change of the lattice parameter $a$ is shown on the right, followed by 3D and 2D electron density maps and a plot of the volumes adsorbed versus the time of adsorption. Selected data from this summary are shown in the main text. 

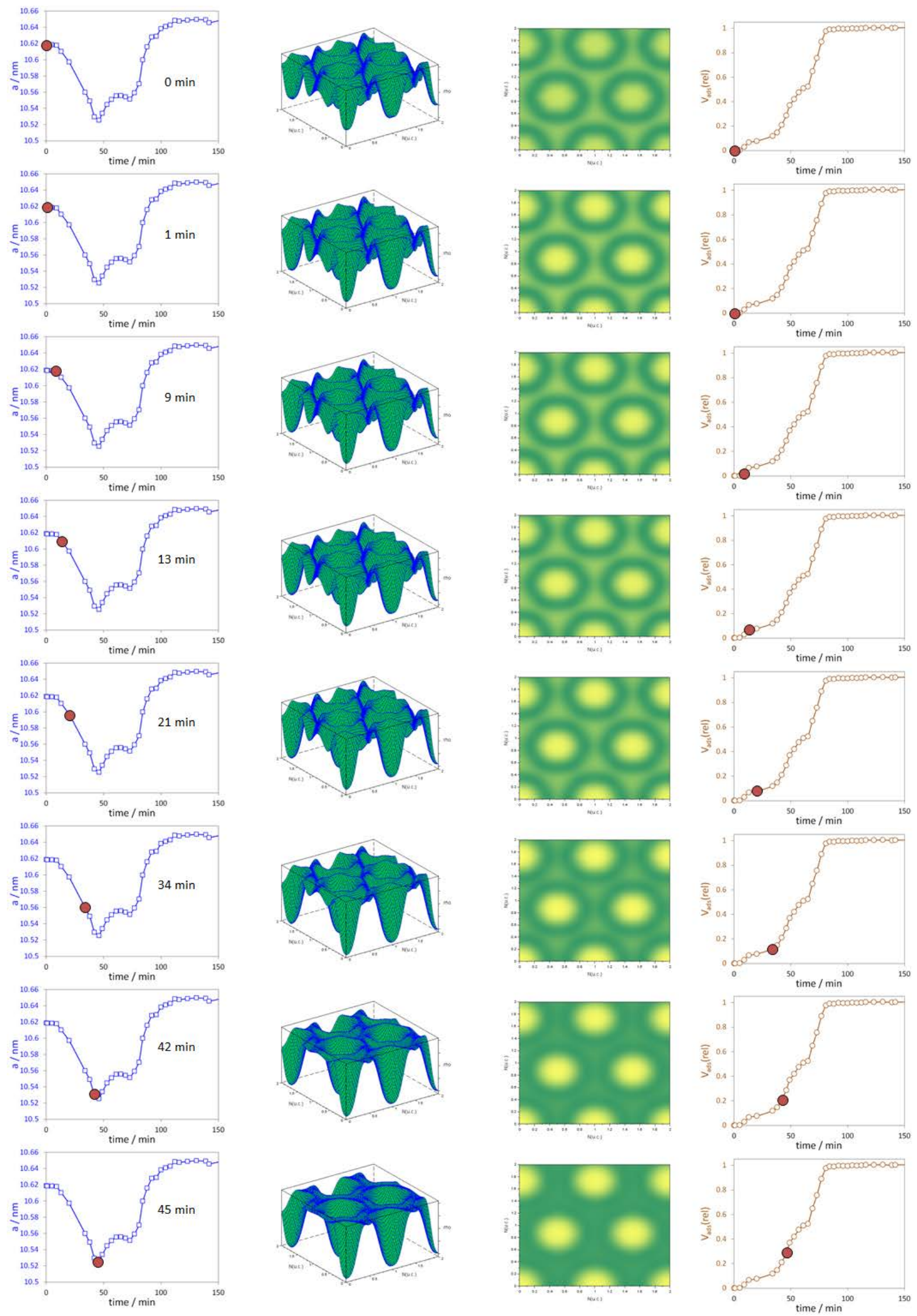

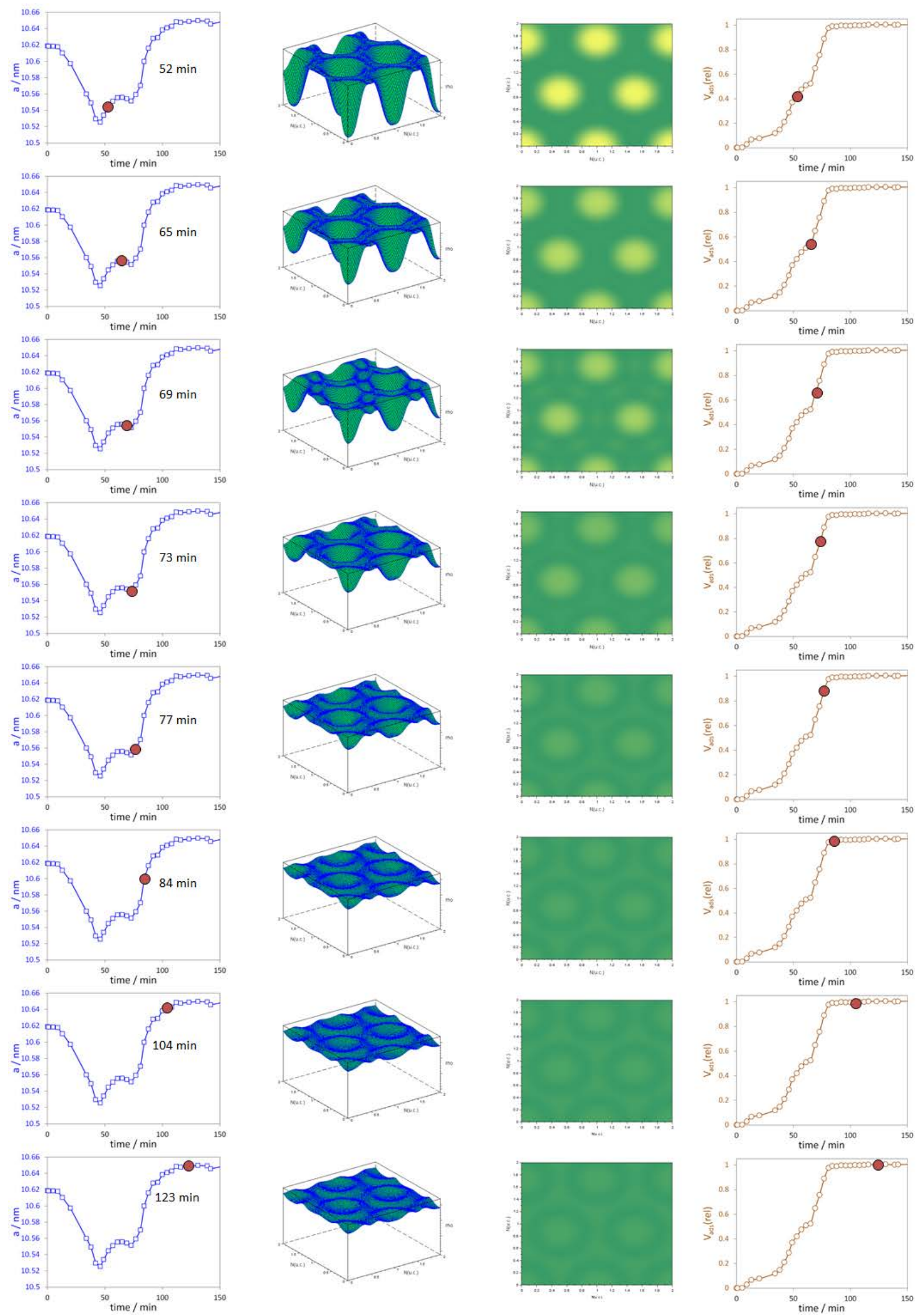


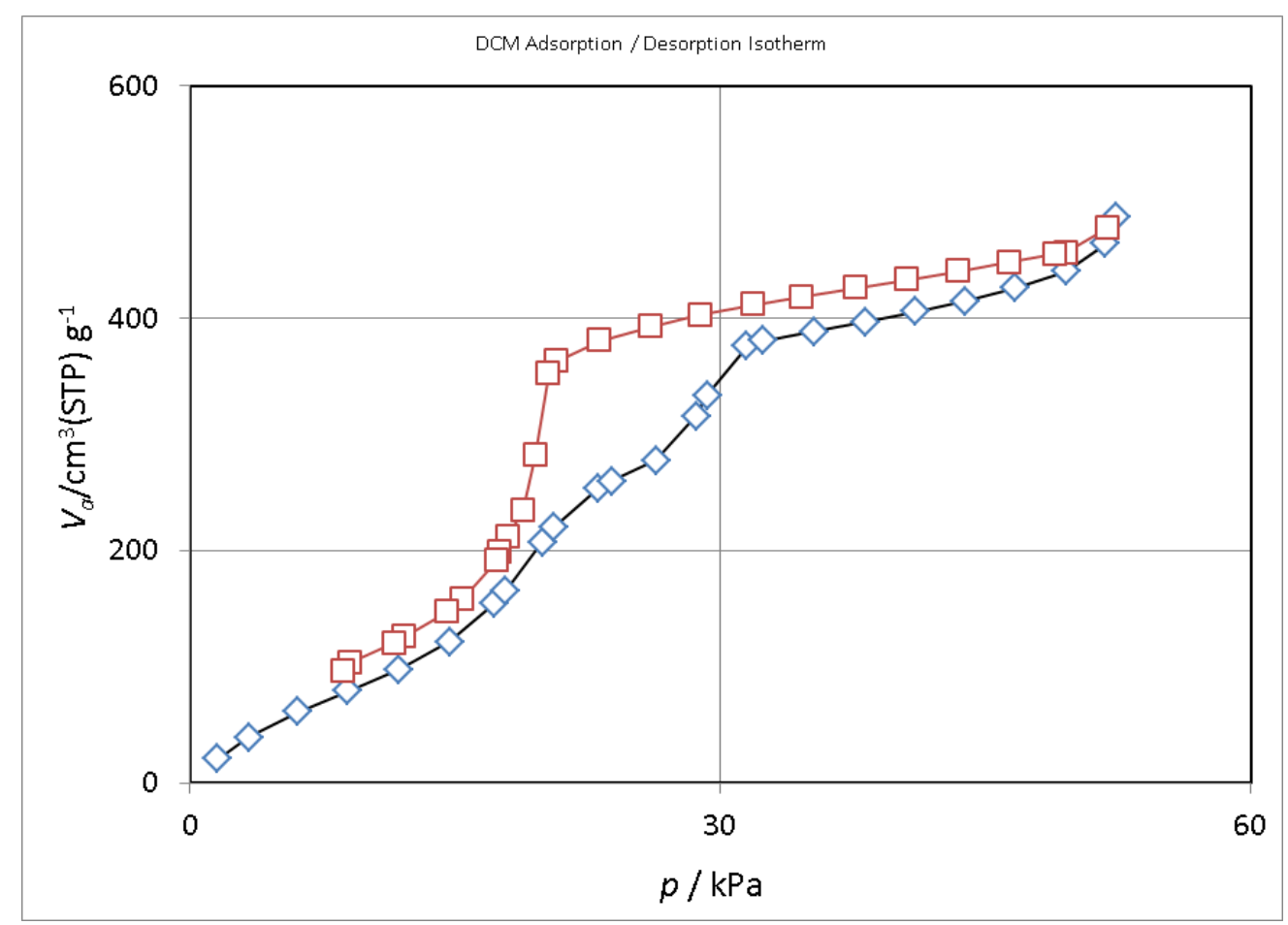

Figure S8. DCM equilibrium adsorption-desorption isotherm measured at $25^{\circ} \mathrm{C}$ (saturation pressure $58.109 \mathrm{kPa}$ ). The data have been recorded on a Belsorp-Max (BEL Instruments, Japan) at Rubotherm (Bochum). Prior to measurement, the sample was activated at $200^{\circ} \mathrm{C}$ for $10 \mathrm{~h}$. (activation at $200^{\circ} \mathrm{C}$ for $10 \mathrm{~h}$ ). 